\title{
MANAGEMENT OF HEPATOCELLULAR CARCINOMA: A STUDY ON 240 PATIENTS IN A SINGLE REFERRAL CENTER
}

\author{
Krasimir Ivanov
}

\author{
Medical University of Varna
}

Epidemiology of hepatocellular carcinoma (HCC) is changing globally and trends show an increasing morbidity rates in Northern, Central and Eastern Europe and North America but a slow decline in Japan, Korea and China. The burden of this important complication of chronic advanced liver disease will further increase in the next 10 years. Several reviews focus on a broad spectrum of HCC clinical management worldwide, including surveillance of patients with cirrhosis thus establishing proper HCC diagnostic and therapeutic options.

The study of I. Ivanova et al. involves patient data from a single referral center in St. Marina University Hospital of Varna. It is the first report of the results of a project entitled 'Hepatocellular Carcinoma - a Current Diagnostic and Therapeutic Approach'. The study achieves its goal to present the results from this multidisciplinary HCC treatment.

This is one of the three largest cohort studies in Bulgaria so far. The paper reports 240 HCC patients during an 11-year period (from 2006 to 2016) in the Clinic of Gastroenterology, St. Marina University Hospital of Varna. These results aim at evaluating the risk factors for HCC and etiology of chronic liver disease, the characteristics and severity of underlying liver disease, the methods for HCC diagnosis and tumor burden as well as staging and treatment methods.

The analysis of the risk factors for HCC and etiology of chronic liver disease shows that chronic hepatitis- $\mathrm{B}$ virus (HBV) infection has been registered in $40.4 \%$ and chronic hepatitis- $\mathrm{C}$ virus (HCV) infection - in $25.8 \%$ of HCC patients. There are two cases with HBV/HCV co-infection and four had HBV/hepatitis-D virus (HDV) co-infection. The other rare etiologic factors for chronic liver disease in our cohort are the following: Wilson's disease in three, autoimmune hepatitis - in two and non-alcoholic fatty liver disease (NAFLD) in one patient. Unknown etiology has been registered in 28 patients, as 10 of them (36\%) have presented with a marker for exposure to HBV (anti-HBc positive antibodies). Despite the common understanding of the link between liver cirrhosis and HCC, only $82 \%$ of HCC patients have developed this pathology. HCC is the first complication of chronic liver disease in $2 / 3$ of cases $(n=156)$. One limitation of the study when comparing laboratory results is the lack of similar cohort of patients without malignant transformation.

The diagnostic algorithm includes various imaging and biopsy modalities. The primary diagnosis of HCC is based on at least two imaging methods in 133 cases. In any other patients, the imaging method is combined with at least one ultasonography- or computed-tomography-guided biopsy of the hepatic lesions. The patients with very early and early HCC stage account for $13 \%$ of all HCCs.

The treatment modalities encompass every current modality available such as surgical resection (in 23\%), radiofrequency ablation (in 5\%) and transarterial chemoembolization (TACE) (in 2\% of the cases). Systemic therapy, predominantly with Sorafenib, has been introduced to $17 \%$ of the patients.

The significance of ultrasound surveillance in liver cirrhosis patients is confirmed with the large percentage of diagnosed tumors - 51\%. In contrast, most HCCs are detected at stage D, mainly due to advance baseline liver cirrhosis.

In summary, findings from this study confirm that surveillance with ultrasound and tumor markers like alpha-fetoprotein (AFP) among patients with advanced liver disease may increase HCC detection rate and achieve longer patient survival rate. 